\title{
Benjamin Taunay, Le Tourisme intérieur chinois : approche géographique à partir de provinces du sud- ouest de la Chine
}

Thèse de doctorat en géographie, université de La Rochelle, dirigée par Patrice Cosaert et Isabelle Sacareau

(thèse soutenue le 27 novembre 2009)

\section{Benjamin Taunay}

\section{(2) OpenEdition}

\section{Journals}

Édition électronique

URL : http://journals.openedition.org/tourisme/330

DOI : 10.4000/tourisme.330

ISSN : 2492-7503

Éditeur

Éditions touristiques européennes

Édition imprimée

Date de publication : 1 juin 2010

Pagination : 81-84

ISSN : 2109-5671

Référence électronique

Benjamin Taunay, «Benjamin Taunay, Le Tourisme intérieur chinois : approche géographique à partir de provinces du sud-ouest de la Chine », Mondes du Tourisme [En ligne], 1 | 2010, mis en ligne le 30 septembre 2015, consulté le 22 septembre 2020. URL : http://journals.openedition.org/tourisme/330 DOI : https://doi.org/10.4000/tourisme.330

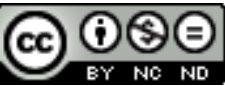

Mondes du tourisme est mis à disposition selon les termes de la licence Creative Commons Attribution - Pas d'Utilisation Commerciale - Pas de Modification 4.0 International. 


\title{
Le tourisme intérieur chinois :
}

\section{approche géographique à partir de provinces du sud-ouest de la Chine}

\author{
Thèse de doctorat en géographie, université de La Rochelle, \\ dirigée par Patrice Cosaert et Isabelle Sacareau (soutenue le 27 novembre 2009) \\ B E N J A M IN TA UNAY_ [benjamin.taunay@gmail.com]
}

$C^{2}$ e texte souhaite revenir sur les principales interrogations proposées dans un travail de thèse ${ }^{(1)}$ portant sur les pratiques des "touristes intérieurs" chinois et cherchant à mieux comprendre les logiques de production des espaces touristiques destinés à ces touristes non occidentaux. Le tourisme intérieur est un thème encore très peu traité par la littérature géographique, et l'espace chinois ne fait pas exception à ce constat. Les seules études disponibles ont toutes des visées économiques et/ou d'aménagement. Ce travail de thèse s'est donc davantage orienté dans une perspective de géographie culturelle, en axant l'analyse sur les pratiques spatiales des touristes et les fondements socioculturels de celles-ci. Cette exploration du tourisme intérieur chinois a été principalement effectuée dans le sudouest du pays, région où nous pouvons maintenant mieux considérer, d'une part, les pratiques des touristes chinois et leur manière de regarder les espaces naturels et urbains et, d'autre part, les transformations urbaines induites par le tourisme, utilisé comme outil de développement dans des régions pauvres.

\section{ÉTAT DES LIEUX DU TOURISME INTÉRIEUR CHINOIS}

Nous avons tout d'abord cherché à caractériser le tourisme intérieur en nous interrogeant sur sa réalité numérique, ses fondements, ainsi que sur les acteurs qui l'ont développé - l’État, en particulier. De cette analyse, il est ressorti que cette forme de tourisme méconnue dépasse considérablement celle des internationaux présents dans le pays. Les statistiques surestiment la forme internationale du tourisme en Chine, mais le tourisme intérieur, et c'est là le point fondamental, fournit à ce secteur d'activité la majeure partie de ses revenus nationaux. Depuis son apparition "moderne" à la fin des années 1970 et sa reconnaissance par l'État environ dix ans plus tard, le tourisme intérieur n'a cessé de bénéficier d'encouragements de la part du gouvernement. À la fin des années 2000, environ 300 millions de touristes chinois sillon- nent leur pays à la recherche de l'altérité. Ce chiffre pourrait être revu à la hausse si l'analyse englobait les touristes provenant de Hong Kong et de Macao, mais les formes, les pratiques, ainsi que les représentations qui guident ces individus en Chine continentale diffèrent de celles des personnes nées et vivant encore en République populaire, excluant de fait les ressortissants des régions administratives spéciales, et à plus forte raison ceux de Taïwan, de l'étude du tourisme intérieur.

Autant les touristes chinois sont nombreux au sein de leur pays, autant les fondements de ce tourisme sont anciens. Il existait des formes pré-touristiques dès le $\mathrm{XV}^{\mathrm{e}}$ siècle, bien avant celles qui donnèrent plus tard naissance au tourisme en Europe. Pourtant, il n'y a pas eu de développement touristique en Chine avant la fin du XX siècle, d'abord parce que le pays n'a pas connu la séparation entre temps de travail et temps de repos, engendrée par la Révolution industrielle sur le vieux continent. Le tourisme intérieur repose donc seulement sur la 
couche urbanisée de la Chine contemporaine, une population qui recherche, au quotidien comme lors de ses loisirs et de ses vacances, une certaine forme de "modernité”. Les travaux récents de quelques anthropologues s'étant penchés sur la question le prouvent : les lieux développés pour ces touristes sont profondément transformés et aménagés pour la commodité des visiteurs, même s'il s'agit d'un village de "minorités" situé dans une zone rurale. Le regard ne se porte pas sur "l'authenticité" des constructions ni sur l'origine des spectacles qu'ils observent, mais bien sur une mise en scène qui mêle la tradition à la consommation. Une géographie du tourisme intérieur, soulignant la prédominance des touristes originaires des principales villes parmi les plus développées du pays, ainsi que l'exposé de la recherche au quotidien d'un certain confort de la part de ces mêmes individus, explique en partie cette recherche de "modernité". Elle explique également qu'une population de touristes plus individuels fasse son apparition depuis ces grands centres urbains, et se dirige avec un autre regard et d'autres aspirations que leurs aînés vers les hauts lieux touristiques, voire vers d'autres sites moins connus.

Au terme de cette description du tourisme intérieur, nous avons jugé fondamental de mettre en valeur la dynamique des acteurs qui sous-tend ce phénomène. L'État est bien le principal protagoniste du tourisme intérieur, depuis le lancement de lieux à la fin des années 1970, jusqu'à aujourd'hui, où il continue de donner des directives générales, scrupuleusement appliquées à l'échelle locale. Le manque de littérature spécialisée nous a conduit à utiliser des cas particuliers pour éclairer des stratégies plus générales. Grâce à l'analyse successive des mises en tourisme de Guilin puis de Beihai, deux villes situées dans la région autonome zhuang du Guangxi ${ }^{(2)}$, l'exposé a fait apparaître deux scénarios, deux trajectoires urbaines liées à l'activité touristique : l'argument des paysages ${ }^{(3)}$ a été placé en premier plan à Guilin, tandis que le développement du tourisme à Beihai a été un prétexte pour développer un littoral marginal qui avait du mal à se sortir d'une pauvreté ancienne. Ces deux exemples ont, au-delà de leur aspect descriptif, permis de présenter les deux aspects centraux de notre travail ; ils ont aussi montré que l'influence de l'État, aussi considérable soit-elle, n'est pas obligatoirement le gage d'une mise en tourisme réussie. La valeur des lieux intervient dans le choix des touristes au moment de partir pour telle ou telle destination : la plage de Beihai demeura au stade de l'échec cuisant tant qu'elle ne fut pas transformée pour les goûts et les représentations des touristes intérieurs.

\section{LES PRATIQUES SPATIALES DES TOURISTES INTÉRIEURS}

L'analyse s'est, dans la deuxième partie du mémoire, portée plus spécifiquement sur les pratiques spatiales des touristes intérieurs, ainsi que sur une plongée dans les représentations et les valeurs qui guident ces comportements et déplacements dans l'espace. Pour cela, une comparaison quasi constante avec les pratiques des touristes occidentaux, en groupe et de manière plus individuelle, a été notre fil conducteur. Il ressort de cet examen que de profondes différences séparent ces deux formes de tourisme dans le pays. Pour en témoigner, la démonstration a d'abord fait appel à une approche statistique, quantitative, qui a permis d'extraire des caractéristiques générales des pratiques chinoises, au travers des cas particulier de Guilin principalement, de Beihai dans une moindre mesure. Dans les deux cas, s'il est évident qu'un certain conformisme règne sur le tourisme intérieur, il n'en reste pas moins que des pratiques plus individuelles se font jour. Celles-ci sont d'abord le fait d'une variable générationnelle, plus que de l'origine géographique des touristes. Dans un pays où l'histoire récente reste tourmentée, l'âge est donc un aspect à prendre en compte dans les analyses touristiques qui suivront.

Les pratiques liées à la nature et au paysage restent, elles, profondément marquées par l'origine des touristes. Dans différentes régions du Guangxi en général, à Guilin et ses environs en particulier, les touristes intérieurs et les touristes occidentaux portent un regard très différencié sur les sites naturels qu'ils visitent : chez les Chinois, c'est la forme - et la signification imagée de celle-ci - qui prime sur la connaissance ; chez les Occidentaux, en revanche, c'est l'intérêt pour l'aspect scientifique ainsi qu'un regard fondé sur le romantisme qui l'emportent. Le paysage ne prend donc sens et forme qu'en fonction de celui qui le regarde, et il en découle des territoires touristiques assez différents : à Longji par exemple (dans le nord-ouest du Guangxi), les touristes chinois dépassent rarement le village animé alors que les Occidentaux préfèrent s'isoler au sommet des terrasses, voire dans les chemins sinueux qui relient les 
hameaux entre eux. Il faudrait là encore nuancer cette affirmation selon les différentes générations de touristes, les pratiques des plus jeunes Chinois ressemblant parfois à celles des backpackers occidentaux. À une dissemblance près : certains lieux visités par les premiers ne sont souvent pas connus des seconds.

Les villes sont en revanche des espaces fréquentés par les deux populations touristiques; toutes les générations s'y retrouvent mais, ici comme ailleurs, toutes n'y témoignent pas des mêmes pratiques. Les Chinois souhaitent y voir un lieu moderne, spécialement ceux qui ont connu les rigueurs de la Révolution culturelle et pour qui la "campagne" évoque son lot de souffrances et d'aversions. Pour eux, un séjour touristique ne saurait se passer hors des plus grandes villes, à l'exception d'une croisière sur le fleuve $\mathrm{Li}^{(4)}$ parce qu'ils y voient la quintessence du paysage. Pour les autres, c'est aussi une vision édulcorée de la réalité paysanne qui plaît, montrant que l'expérience traumatisante de la Révolution culturelle a profondément marqué les relations de la société chinoise, dans sa globalité, avec la campagne. Les parcs en ville font donc recette, à la grande satisfaction de l'État - qui y voit un moyen de construire une identité nationale, ostensible dans l'agencement des circuits et dans les aménagements en partie dédiés à la "gloire de la Chine" (à Guilin en particulier). Modernité, distractions ("entertainment") et construction de l'identité nationale sont complétées par une certaine recherche de la sociabilité dans les lieux touristiques : sur la plage de Beihai, les touristes se massent d'abord dans un cœur “renao" (bruyant et animé), où les aménagements sont nombreux quoique jugés insuffisants par les touristes.

\section{LES TRANSFORMATIONS URBAINES POUR LE DÉVELOPPEMENT}

Dans la dernière partie de ce travail, la réflexion s'est portée sur l'utilisation du tourisme intérieur comme d'un outil de développement dans des régions anciennement en marge voire, pour certaines, toujours actuellement dans ce contexte défavorable. Il ressort de cette analyse que les espaces anciennement touchés par le tourisme ont été profondément transformés, tout d'abord au niveau des nouveaux et nombreux aménagements qui ont été construits au cœur même des villes. Même si les premières transformations n'étaient pas orientées vers le tourisme intérieur, un changement de stratégie s'est rapidement produit, pour épouser la réalité de la situation touristique dans le pays : dès le milieu des années 1990, le tourisme international a été supplanté numériquement par son homologue intérieur.

L'État, relayé à l'échelle sousprovinciale par les gouvernements des différentes municipalités, a massivement investi, allant souvent jusqu'à déplacer le centreville pour le conformer aux goûts des touristes intérieurs. Les aménagements doivent en effet témoigner du progrès, de la modernité, même quand il s'agit de lieux patrimoniaux, créant ainsi des espaces où le vernaculaire disparaît rapidement sous la reconstruction "inauthentique". Place est également faite aux centres commerciaux ainsi qu'aux longues rues commerçantes, ce qui forme des villes aux configurations nouvelles qui restent à étudier.

L'objectif initial de transformer les villes grâce au tourisme, d'en faire des espaces modernes et développés, mais aussi de permettre à ces espaces de prendre une part active dans le commandement économique à l'échelle sous-provinciale et parfois provinciale, a donc été en partie atteint. L'objectif actuel avoué de Guilin est, par exemple, de diversifier son économie, encore aujourd'hui en grande partie centrée sur le tourisme ; celui de Beihai, de continuer à se développer avec le tourisme. Outre que les scénarios diffèrent aujourd'hui comme autrefois, leur base commune fut celle de se propager, plusieurs décennies après le choix de l'insertion du tourisme dans les plans d'urbanisme, sur un espace qui s'est étendu, s'est densifié et s'est rempli d'une nouvelle population attirée par le développement touristique et économique. Les espaces urbains stricto sensu, mais aussi les espaces périphériques aux villes, ont été influencés par ces transformations : Guilin se retrouve aujourd'hui au cœur d'une vaste périphérie touristique où des transformations se produisent à leur tour ; même Beihai, hier en marge et sans "valeur" touristique, se trouve en position de définir une ceinture touristique depuis qu'elle est devenue une ville d'importance à l'échelle de la province du Guangxi.

Le tourisme intérieur se diffuse donc actuellement là où, il y a seulement quelques décennies, personne n'aurait parié sur un hypothétique développement. Même si le village de Yangshuo est connu depuis plusieurs centaines d'années comme étant la perle des paysages de la région de Guilin, il ne s'est vraiment ouvert au tourisme 
que tardivement, son essor ayant été longtemps restreint par un gouvernement de Guilin soucieux de contrôler le développement de ses périphéries. Il constitue aujourd'hui un exemple de choix pour analyser la diffusion du tourisme intérieur à une échelle régionale : ancienne périphérie, le village s'est mué en petite ville qui contrôle désormais sa propre ceinture touristique, et qui est accessible directement par la route depuis Canton (à $600 \mathrm{~km}$ à l'est de Yangshuo), alors qu'autrefois une étape à Guilin était de mise. Fruits d'une nouvelle et récente volonté de l'État de développer un tourisme plus "local", Yangshuo et d'autres lieux se retrouvent aspirés dans la spirale vertueuse du développement économique impulsée grâce au tourisme. Même les plus petits interstices pauvres essaient, parfois de manière autonome, d'attirer les touristes pour sortir de leur isolement, en particulier les régions à l'ouest du Guangxi. Grâce au tourisme intérieur, certaines parties du Guangxi commencent parfois à trouver un second souffle, prouvant donc que le tourisme est un outil de développement qui prend de l'ampleur à l'échelle du sudouest chinois.

À l'issue de ce travail de recherche, il subsiste toutefois de nombreuses zones d'ombre dans la connaissance de cette forme de tourisme. Eu égard à la taille de ce pays, il faudrait évidemment densifier l'approche empirique entamée ici, autant sur les pratiques que sur les lieux, en l'élargissant à d'autres parties de la Chine continentale. En effet, dans un autre cadre (le Sud-Ouest représente seulement $8,4 \%$ de la superficie du pays), le sujet aurait peut-être été différent. Il serait également intéressant de poursuivre l'analyse de la thématique "Chine, ville, tourisme" dans d'autres villes du pays, en particulier dans des espaces urbains plus vastes et plus denses que les cas traités ici ; et cela à de multiples échelles spatiales - de la municipalité à la dimension infraurbaine, voire à celle des différents aménagements -, dans un objectif à venir de classification d'un lieu en fonction des stratégies suivies, de sa trajectoire urbaine et de sa mise en tourisme, de sa situation au sein du pays, de sa taille, etc. Il faudra y revenir.

( I) Benjamin TAUNAY, Le Tourisme intérieur chinois: approche géographique à partir de provinces du sud-ouest de la Chine, thèse de doctorat en géographie, université de La Rochelle, novembre 2009.

(2) Respectivement au nord-est et au sud-est de la province. Guilin (700 000 habitants) est internationalement connu pour ses paysages naturels alors que Beihai (300 000 habitants) est une ville dont la renommée n'est que nationale, voire régionale.

(3) La culture chinoise considère Guilin comme la quintessence des paysages (au sens de "montagne et eau qui court") de la Chine. Ce qui s'exprime dans l'adage littéraire: "Les plus beaux paysages sont à Guilin."

(4) Le site le plus connu de la région de Guilin. Il est inscrit sur la liste du patrimoine mondial de l'Unesco. 